\title{
A Structure Model of Clinical Nurses' Silence on Patient Safety
}

\author{
Han, Jeongwon ${ }^{1)}$
}

\section{1) College of Nursing, Kosin University, Busan, Korea}

Purpose: The aim of this study was to establish a hypothetical model on silence regarding patient safety and to verify the model's goodness of fit and hypotheses. Methods: The participants in this study were 330 registered nurses working in tertiary hospitals with over 300 beds. Data were collected between July 1, and August 30, 2017, from nurses who agreed to participate. A covariance structure analysis was performed. Results: The model of fit index was $x^{2}=59.54$, normed $x^{2}=2.29, \mathrm{GFI}=.97, \mathrm{AGFI}=.93$, SRMR=.05, NFI=.99, CFI=.95 and RMSEA $=05$. The organizational culture had an influence on patient safety motivation $(\beta=.26, p=.003)$ and attitude $(\beta=.43$, $p<.001)$. RN-MD collaboration had an influence on patient safety motivation $(\beta=.33, p<.001)$, attitude $(\beta=.35$, $p<.001)$, and patient safety silence $(\beta=-.17, p=.026)$. Supervisory trust had an influence on patient safety motivation $(\beta=.26, p<.001)$, attitude $(\beta=.12, p=.036)$, and patient safety silence $(\beta=-.23, p=.002)$. Patient safety motivation had an influence on patient safety silence $(\beta=-.33, p=.006)$. The model of patient safety silence explained $36.0 \%$ of the variances. Conclusion: This study is meaningful in that it provides basic data for nursing education and program development for rejecting patient safety silence.

Key Words: Collaboration, Culture, Nurse, Safety, Trust

*This work was supported by the Ministry of Education of the Republic of Korea and the National Research Foundation of Korea (NRF-과제번호)(NRF-2017S1A5A8020631).

Received Nov 3, 2017 Revised Dec 30, 2017 Accepted Feb 12, 2018

Corresponding author: Han, Jeongwon

College of Nursing, Kosin University

262 Gamcheon-ro, Seo-gu, Busan 49267, Korea

Tel: +82-51-990-3986, Fax:+82-51-990-3986, E-mail: hjw0721@naver.com 


\section{INTRODUCTION}

There were an estimated 44,009,900 cases of patient safety incidents in the United States in 2015 with associated economic costs to the nation between 17 to 29 billion dollars and in Australia, approximately 25,000 people were estimated to have permanent disabilities due to safety accidents in hospitals [1]. According to the $2013 \mathrm{Na-}$ tional Health Insurance Statistical Yearbook, Korea had approximately 19,013 deaths from preventable adverse events among 6,420,118 hospitalization cases [2]. When these figures were compared to the 2013 data from National Statistics Office of Korea, including 15,906 suicide deaths in Korea, 6,361 deaths from automobile accidents, and 2,144 deaths from falling, the costs to citizens from patient safety accidents are regarded as socially detrimental [3].

As patient safety accidents constitute an important problem that threatens the health of citizens, medical institution accreditation was introduced to improve the quality of medical institutions in favor of patient safety; on January 28, 2015, the Patient Safety Act was passed into law [4]. It is important to focus on the legislation of autonomous reporting systems among the key contents of the Patient Safety Act. Article 14, Section 2 of the Patient Safety Act proposes that if an individual causing a patient safety accident engages in autonomous reporting, the relevant administrative dispositions under the Medical Service Act and other Acts or subordinate statutes related to health and medical services may be mitigated or exempted; this includes sections to protect the confidentiality of autonomous reporting and whistleblowing individuals [5]. In particular, the judicial branch focuses on autonomous reporting related to patient safety accidents. The autonomous reporting is the most accurate method of identifying the status of such accidents as it it allows organizations to incentivize individuals to proactively participate in event perception, quality improvement processes, and feedback processes and works well to change the attitudes and actions of individuals regarding safety accidents [6].

However, realistically, it is difficult for medical professionals to effectively engage in autonomous reporting in actual clinical settings. This is because most medical professionals maintain silence regarding patient safety accidents [7]. Evaluating the behavior of silence adopted by medical professionals on the matter of patient safety accidents based on the Social Exchange Theory, humans act on their own decisions in situations of conflict but ultimately internalize the desire to maximize gains and minimize risk. As such, if the perceived rewards are less than the perceived costs or punishment, individuals tend to choose actions to protect themselves [8]. In other words, the behavior of silence by medical professionals regarding patient safety accidents is sometimes caused by the lack of knowledge or information on the reporting of such incidents. However, most medical professionals fear punishment or loss of their jobs and promotion opportunities and thus engage in the purposeful and strategic behavior of avoiding reporting patient safety accidents [9].

The behavior of silence by members of an organization is caused by a complex mix of organization- and individual-level influences. At the organization level, these factors include authoritative and hierarchical structure, topdown decision-making processes, unfairness of the organization, and the ethical climate of the organization. At the individual level, these factors include the characteristics of the members as well as relationships with supervisors and peers [10]. Particularly, given the situations of hierarchical organizational culture or poor relationships with supervisors, members of organizations tend to take a negative attitude toward freely sharing their opinions or other innovative behaviors that lead to positive results for the organization [11]. Applying the theory by Morrison [9], the patient safety silence of nurses is caused by the lack of incentive for, and interest in patient safety by nurses. Continuous interest in and incentivization for patient safety by the members of the organization are supported by an atmosphere of mutual support as well as a support structure that makes responses to such patient safety accidents effective [12]. Moreover, according to the existing literature on the nurses and doctors from oncology wards in Switzerland [13], the medical professionals cited incentives to present their opinions on patient safety as being the most important factor in rejecting patient safety silence; some of the hindering factors included hierarchical organizational structures and negative results associated with reporting patient safety accidents.

With regard to the existing foreign literature on patient safety silence, Martinez et al.[14] argued that not only clinical nurses but also doctors in the U.S. must reject silence towards patient safety, emphasizing that this silence begins with indifference towards patient safety and arguing for raising the interest in and attitude toward patient safety by medical professionals. Ullstrom et al.[15] studied nurses and doctors in Sweden regarding the difficulties they experienced with respect to patient safety silence and concluded that medical professionals desire to reject silence on patient safety, and this was impeded by the lack of organizational resources and peer-level support structure. On the other hand, existing literature on patient safety among nurses in Korea generally relates to the level of 
perception of patient safety by medical professionals [16], attitudes toward patient safety [17], and factors influencing safety engagement among nurses [18] with a general lack of studies on patient safety silence by medical professionals as well as tools to measure the level of silence by nurses.

Patient safety accidents constitute an important social problem that is directly related to the health of citizens, and the prevention of accidents has become very important. However, the general lack of autonomous reporting by medical professionals presents limitations for building a comprehensive response to patient safety. As nurses constitute a majority of the medical professional population and are at the forefront of providing healthcare services, rejecting the silence of nurses on patient safety is a very important aspect in preventing patient safety accidents. However, the lack of domestic studies on nurses' patient safety silence compared to overseas studies presents limitations in setting up patient safety systems and human resource management strategies for medical institutions. Moreover, to activate the autonomous reporting system that has been rolled out at a national level, it is important to conduct studies on patient safety silence of nurses. As such, the authors of this study sought to confirm the factors influencing patient safety silence among clinical nurses and confirm their interrelationships, the factors which promote nurses rejecting the behavior of silence on patient safety, and provide basic data for reactivating the autonomous reporting systems within the hospital organizations.

As such, the specific research objectives were as follows.

- Construct a hypothetical model to explain the patient safety silence of nurses.

- Verify the goodness of fit of the hypothetical model in relation to the actual data and propose a modified model that explains and predicts the patient safety silence of nurses.

- Identify the direct and indirect effect coefficients of factors influencing nurses' patient safety silence and determine the level of correlation between the factors.

\section{METHODS}

\section{Study Design}

In this study an attempt was made to establish a hypothetical model of the organizational culture perceived by nurses, RN-MD collaboration, supervisory trust, patient safety motivation, patient safety attitude, and patient safety silence and perform a covariance structure analysis to verify the model's goodness of fit and hypotheses (Figure 1).

\section{Study Participants}

The accessible population of this study was set as nurses working in tertiary hospitals with more than 300 beds in Seoul Metropolitan area, Gyeonggi-do, Busan Metropolitan area, and Changwon city. Particularly, the participants in this study were permanently employed nurses working in the department directly related to patient safety. Nurses working in administrative departments, who were less related to patient safety, were excluded from the study. For this study, 330 nurses who consented to participate and gave their written consent in the form of signatures were selected. The appropriate sample size in a structural equation varies among scholars; however, according to the study by Hair et al.[19], a sample size between 150 and 400 individuals is appropriate for structural equations. As such, the sample size of this study can be considered appropriate.

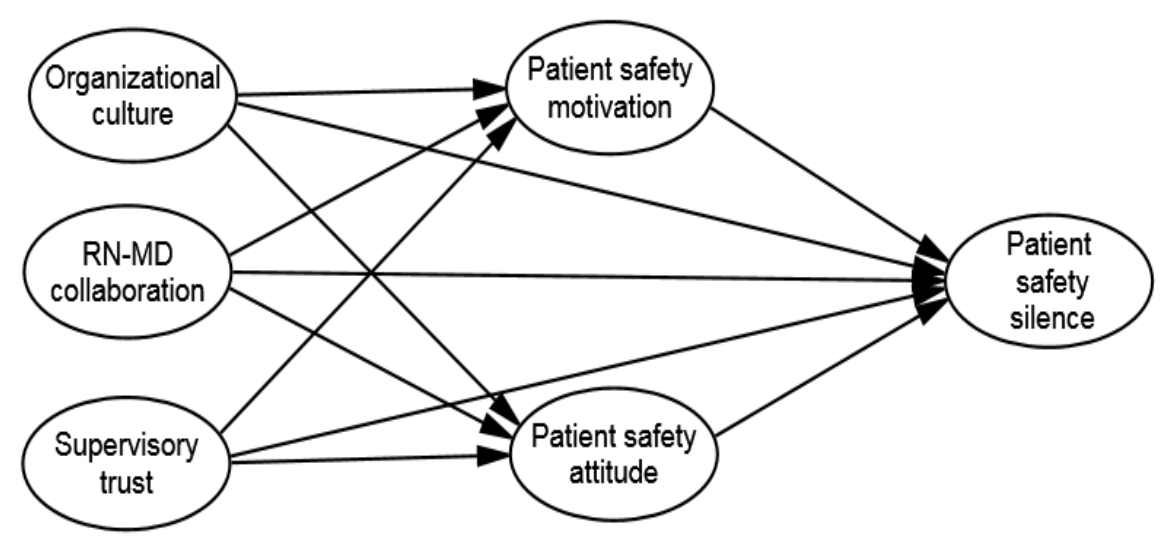

Figure 1. Hypothetical model. 


\section{Measurements}

A confirmatory factor analysis (CFA) was performed for each tool in order to verify the validity of the research tools first, and then the hypothetical model was verified. Questions with a factor loading of at least .60 were selected. Normed $x^{2} \leq 3.00$, adjusted goodness of fit index (AGFI $\geq .90$ ), goodness of fit index (GFI $\geq .90)$, comparative fit index ( $\mathrm{CFI} \geq .90)$, standardized root mean square residual (SRMR $\leq .05)$, root mean square error of approximation (RMSEA $\leq .10)$, and normed fit index (NFI $\geq .90)$ were considered the optimal standards for the model goodness of fit of CFA [19]. The convergent validity was measured by factor loading, construct reliability (CR), and average variance extracted (AVE).

\section{1) Organizational Culture}

Organizational culture refers to the type of values and beliefs that have been accumulated by the organization members in the history of the organization. For this study, the tool used was a measurement tool developed by Quinn and McGrath [11] and modified by Choi [20] for use with hospital workers. The measurement tool for organizational culture is composed of 12 questions with 4 subfactors (5-point scale); there are 3 questions each on innovative organizational culture, relational organizational culture, work-oriented organizational culture, and hierarchical organizational culture with higher scores indicating positive organizational culture. The evaluation of the model goodness of fit of CFA showed that normed $x^{2}=$ 2.26, GFI $=.95$, RMSEA $=.05$, SRMR $=.03, \mathrm{NFI}=.95$ and $\mathrm{CFI}=$ .97 , respectively, which are considered good. Factor loading was between .70 and $.87, \mathrm{CR}$ ranged between .85 and .93, while AVE was between .66 and .81. Cronbach's $\alpha$ was $.66 \sim .80$ in a study by Choi [20]; in this study, it was .78 .88.

\section{2) RN-MD Collaboration}

RN-MD collaboration was measured using a tool developed by Ushiro [21], which was translated into Korean and modified. The RN-MD collaboration measurement tool is composed of 3 sub-factors (decision making on treatment, sharing of patient's information, and RN-MD relationship) and 27 questions (5-point scale) with higher scores indicating higher levels of RN-MD collaboration. The evaluation of the model goodness of fit of CFA showed that normed $x^{2}=2.40, \mathrm{GFI}=.90, \mathrm{RMSEA}=.10$, $\mathrm{SRMR}=.04, \mathrm{NFI}=.92$ and $\mathrm{CFI}=.95$, respectively, which are considered good. When one question with less than 60 factor loading was excluded, the factor loading was between .72 and .92 , CR ranged between .63 and .85 , while
AVE was between .60 and .66. Cronbach's $\alpha$ was .95 in a study by Ushiro [21]; in this study, it was .83 .94.

\section{3) Supervisory Trust}

Supervisory trust refers to the trust that team members develop for their superiors through interactions with them. This study used a tool developed by Cook and Wall [22], which was modified by Lee [23]. The measurement tool for supervisory trust is composed of 6 questions (7-point scale) with higher scores indicating higher levels of trust in their supervisors. The evaluation of the model goodness of fit of CFA showed that normed $x^{2}=1.40, \mathrm{GFI}=.95$, RMSEA $=.05$, $\mathrm{SRMR}=.03, \mathrm{NFI}=.95$ and $\mathrm{CFI}=.96$, respectively, which are considered good. Factor loading was between .78 and .92, CR was .90, while AVE was .60. In a study by Lee [23], Cronbach's $\alpha$ was .84; in this study, it was .93.

\section{4) Patient Safety Motivation}

Patient safety motivation refers to a state in which an individual is motivated to strive for the safety of patients. For this study a tool developed by von Thaden and his colleagues [24], and modified for clinical nurses by Kim [25] was used. The measurement tool for patient safety motivation is composed of 6 questions (5-point scale) with higher scores indicating higher levels of motivation for patient safety. The evaluation of the model goodness of fit of CFA showed that normed $x^{2}=2.19$, GFI $=.97$, RMSEA $=.06$, SRMR= $.04, \mathrm{NFI}=.94$ and $\mathrm{CFI}=.97$, respectively, which are considered good. When two questions with less than .60 factor loadings were excluded, the factor loading was between .63 and $.87, \mathrm{CR}$ was .82 , while AVE was .63. Cronbach's $\alpha$ was .71 in a study by Kim [25]; in this study, it was .75.

\section{5) Patient Safety Attitude}

Patient safety attitude refers to the attitude with which medical professionals perceive situations related to the safety of patients. For this study the Safety attitudes questionnaire short form scale (SAQ) developed by Martinez and his colleagues [14] was utilized. Permission to use the tool was obtained from the original author, and it was translated and back translated by a bilingual professional. The translated questions were verified by a clinical nurse holding a Ph.D. in nursing and two professors of nursing. The calculated item content validity index (item-CVI) was 1.00 for all questions. The SAQ is composed of 2 sub-factors (teamwork and safety) and 13 questions (5-point scale). It was translated into Korean after obtaining the author's approval for tool usage and checking for validity; higher scores indicate higher levels of positive attitude toward patient safety. The evaluation of the model goodness 
of fit of CFA showed that normed $x^{2}=2.45$, GFI $=.95$, $\mathrm{RMSEA}=.07, \mathrm{SRMR}=.05, \mathrm{NFI}=.96$ and $\mathrm{CFI}=.96$, respectively, which are considered good. The factor loading was between .67 and $.88, \mathrm{CR}$ ranged between .91 and .92 , while AVE was between .63 and .67. Cronbach's $\alpha$ was .90 in the study by Martinez et al.[14]; in this study, it was .88 .89.

\section{6) Patient Safety Silence}

Patient safety silence refers to a set of actions with which organizational members maintain silence on situations related to patient safety. For this study the tool was a measurement tool modified and supplemented by Tangirala and Ramanujam [26]. Permission from the original author to use the tool was obtained, and it was translated and reverse translated by a bilingual professional. The translated questions were verified by a clinical nurse holding a Ph.D. in nursing and two professors of nursing. The calculated item-CVI was 1.00 for all questions. It is composed of 5 questions (5-point scale). It was translated into Korean after obtaining the author's approval for tool usage and checking for validity; higher scores indicate higher levels of silence on patient safety. The evaluation of the model goodness of fit of CFA showed that normed $x^{2}=1.16, \mathrm{GFI}=.95, \mathrm{RMSEA}=.06, \mathrm{SRMR}=.04, \mathrm{NFI}=.93$ and $\mathrm{CFI}=.97$, respectively, which are considered good. When one question with less than .60 factor loading was excluded, the factor loading was between .63 and .85 , CR was .90 , while AVE was .70. Cronbach's $\alpha$ was .81 in this study.

\section{Data Collection and Analysis}

To confirm the content validity of the measurement tools used in this study, experts were selected, which included one professor of nursing, one professional with a Ph.D. in nursing who is also a professor of nursing management, and 1 clinical nurse with Ph.D. in nursing, with whom the item-content validity index (item-CVI) was verified. All measurement questions were found to be between 0.80 and 1.00, which confirmed its high validity. Data were collected between July 1, and August 30, 2017, with 330 nurses who agreed to participate and who worked in 1 of 5 tertiary hospitals with more than 300 beds located in the Seoul Metropolitan area, Gyeonggi-do, Busan Metropolitan area, and Changwon City. The questionnaires of 309 participants were utilized in this study. The authors visited the nursing departments of target hospitals and explained the objectives of the study. Consent from each hospital was obtained following research approval by each hospital in order to select nurses meeting the inclusion criteria. Based on the policy of the nursing department, the questionnaires were distributed to and retrieved from the wards, which the participants collectively agreed to through their head nurse.

SPSS/WINdows 24.0 (SPSS Korea Data Solution Inc) and AMOS 16.0 (SPSS Korea Data Solution Inc) was used to analyze the collected data using the methods below. Missing data were treated by using the mean the substitution method. If it was a single observation variable, the factor loading and the variance of the measurement error were fixed as 1 and 0 , respectively, to construct a model. SPSS/WINdows 18.0 was used to analyze the demographic characteristics of the participants as well as the validity and reliability of the study tools. It was also used to verify the measurement model to test the validity of the factors for the construction of the structural equation model of the patient safety silence of nurses. The verification of the measurement model was done using AMOS 16.0 through confirmatory factor analysis. Confirmatory factor analysis refers to the validation of a specific relationship between a question and a factor, as to whether a particular question belongs in a factor. Convergent validity, which refers to verifying whether the multiple tools which measure the same concept match, was measured using factor loading, construct reliability, and AVE. Discriminant validity verifies the extent to which the tools measuring different concepts measure them in a discriminating manner. This was measured using correlation coefficients and $\sqrt{A V E}$ values in this study. To verify the model goodness of fit, methods used were $x^{2}$, normed $x^{2} \leq 3.00$, AGFI $\geq .90$, GFI $\geq .90$, CFI $\geq .90, \quad$ SRMR $\leq .05$, RMSEA $\leq .10$, and NFI $\geq .90[19]$. The values for NFI and CFI, which are relative fit indices, indicate good fit if they are over .90. The RMSEA index, which considers model simplicity, shows very good fit when $<.05$, good fit when $<.08$, average fit when $<.10$, and poor fit when $>.10$. To test the significance of the direct, indirect, and total effects of the variables, bootstrapping was used.

\section{Ethical Considerations}

Data collection began after obtaining approval from the Institutional Ethics Committee of K University (KU IRB 2017-0040-01). Prior to the survey, the participants were provided with written explanations of the study objectives and methods as well as statements that their data would not be used for purposes other than this research, and that subject anonymity and confidentiality would be maintained. Before obtaining participants' consent, they were told that they could reject the survey and withdraw their participation at any time throughout the survey process. 


\section{RESULTS}

\section{Demographic Characteristics of Participants}

The participants in this study were 10 men (3.2\%) and 299 women $(96.8 \%)$ with an average age of 29.94 years (range: $21 \sim 51$ years). In terms of their educational background, 108 had graduated from a 3-year college (35.0\%), 27 from an RN-BSN program (8.7\%), and 143 from 4-year universities $(46.3 \%)$. Participants who answered "yes" to religion were $127(41.1 \%)$, and 182 answered "no"(58.9\%). In terms of marital status, 90 were married (29.1\%), and 219 were single or other $(70.9 \%)$. The average total clinical experience was 72.69 months (range: 1 360 months), with 3.3 years in the most recent department. In terms of title, 260 participants were staff nurses $(84.1 \%)$.

\section{Descriptive Statistics and Correlations of Study Variables}

The descriptive statistics of the study variables are shown in Table 1. The Z scores of skewness (-1.02 0.34) and kurtosis $(-0.32 \sim 1.57)$ of all the variables used in this study did not exceed the critical threshold (1.96) at the significance level of 0.05 proposed by Hair et al.[19], thereby meeting the conditions for normal distribution. ShapiroWilk test $(.97, p=.310)$ was conducted to test the multivariate normality, and it was confirmed that the data was normal for univariate and multivariate tests. It was confirmed that there is no issue in using the maximum likelihood estimation. Normally, if the correlation values between the variables exceed .9 , issues of multicollinearity may be proposed; however, there were no variables with correlation coefficients exceeding 0.9 with other variables, confirming that there were no issues with multicollinearity in this study [19]. Validity testing involved testing for construct validity as abstract construct concepts are often used in studies with structural equation models. Construct validity tests whether the construct concepts have been measured correctly. Confirmatory factor analysis was conducted to test for convergent and discriminant validity. AMOS 16.0 was used for the confirmatory factor analysis, and the estimation method utilized the maximum likelihood estimation. Moreover, the AVE values were .60 .81, exceeding the criteria of .50; content reliability was between .82 and .95 , exceeding the criteria of .70 and confirming the presence of convergent validity. Discriminant validity shows the difference between 2 different latent variables. To evaluate discriminant validity, a comparison of the $\sqrt{ } \mathrm{AVE}$ and the correlation coefficient values between 2 construct concepts were used in this study. The $\sqrt{ } \mathrm{AVE}$ values of latent variables were between .77 and .85 , which were higher than the correlation coefficients of latent variables between -.13 and .58, thereby confirming discriminant validity (Table 1).

\section{Model Goodness of Fit of a Hypothetical Model}

To verify the model goodness of fit, methods such as $x^{2}$, normed $x^{2} \leq 3.00$, AGFI $\geq .90$, GFI $\geq .90$, CFI $\geq .90$, SRMR $\leq .05$, RMSEA $\leq .10$, and NFI $\geq .90[19]$ were used in this study. The model goodness of fit index was found to be as follows: $x^{2}=59.54(\mathrm{df}=26, p<.001)$, normed $x^{2}=2.29$, GFI= $.97, \mathrm{AGFI}=.93, \mathrm{SRMR}=.05, \mathrm{NFI}=.99, \mathrm{CFI}=.95$ and $\mathrm{RMSEA}=$ .05 .

\section{Analysis of a Hypothetical Model}

\section{1) Study Hypothesis}

Table 2 shows the results of testing for path coefficients with the exogenous variables of organizational culture perceived by nurses, RN-MD collaboration, and supervisory trust; mediator variables of patient safety motivation and patient safety attitude; and the variable, result of patient safety silence. The organizational culture perceived by nurses had a positive influence on patient safety motivation $(\beta=.26, p=.003)$. RN-MD collaboration had a positive influence on patient safety motivation $(\beta=.33, p<.001)$.

Table 1. Correlations and Discriminant Validity of the Model

\begin{tabular}{|c|c|c|c|c|c|c|c|c|c|}
\hline \multicolumn{2}{|c|}{ Variables } & \multirow{2}{*}{$\frac{\mathrm{M} \pm \mathrm{SD}}{3.15 \pm 0.53}$} & \multirow{2}{*}{$\frac{\text { Mean range }}{1 \sim 5}$} & \multirow{2}{*}{$\begin{array}{l}\mathrm{X} 1 \\
.85\end{array}$} & \multirow[t]{2}{*}{$\mathrm{X} 2$} & \multirow[t]{2}{*}{$\mathrm{X} 3$} & \multirow[t]{2}{*}{$\mathrm{Y} 1$} & \multirow[t]{2}{*}{$\mathrm{Y} 2$} & \multirow[t]{2}{*}{$\mathrm{Y} 3$} \\
\hline $\mathrm{X} 1$ & Organizational culture & & & & & & & & \\
\hline $\mathrm{X} 2$ & RN-MD collaboration & $3.33 \pm 0.62$ & $1 \sim 5$ & $.50^{*}$ & .79 & & & & \\
\hline X3 & Supervisory trust & $5.04 \pm 1.10$ & $1 \sim 7$ & $.46^{*}$ & $.31^{*}$ & .77 & & & \\
\hline Y1 & Patient safety motivation & $3.05 \pm 0.59$ & $1 \sim 5$ & $.44^{*}$ & $.44^{*}$ & $.38^{*}$ & .79 & & \\
\hline Y2 & Patient safety attitude & $3.07 \pm 0.60$ & $1 \sim 5$ & $.58^{*}$ & $.57^{*}$ & $.39^{*}$ & $.56^{*}$ & .81 & \\
\hline Y3 & Patient safety silence & $2.59 \pm 0.60$ & $1 \sim 5$ & $-.27^{*}$ & $-.26^{*}$ & $-.29^{*}$ & $-.13^{*}$ & $-.18^{*}$ & .84 \\
\hline
\end{tabular}

Note. The bold section: discriminant validity; The non bold section: correlation.

${ }^{*} p<.001 ; \mathrm{CCR}=$ Composite construct reliability; AVE=Average variance extracted. 
Table 2. Effects of Predictive Variables on Endogenous Variables in the Model

\begin{tabular}{llllllll}
\hline Exogenous variables & Endogenous variables & $\beta$ & CR & $p$ & SMC & $\begin{array}{c}\text { Direct } \\
\text { effects }(p)\end{array}$ & $\begin{array}{c}\text { Indirect } \\
\text { effects }(p)\end{array}$ \\
effects $(p)$
\end{tabular}

$\beta=$ Standardized regression weights; $C R=$ Critical ratio; $S M C=$ Squared multiple correlations.

Supervisory trust had a positive influence on patient safety motivation $(\beta=.26, p<.001)$. The model of patient safety motivation explained $26.0 \%$ of the variances. The organizational culture perceived by nurses had a positive influence on patient safety attitude $(\beta=.43, p<.001)$. RN-MD collaboration had a positive influence on patient safety attitude $(\beta=.35, p<.001)$. Supervisory trust had a positive influence on patient safety motivation $(\beta=.12, p=.036)$. The model of patient safety attitude explained $49.0 \%$ of the variances. The organizational culture perceived by nurses had a negative influence on patient safety silence $(\beta=-.17$, $p=.026)$. RN-MD collaboration had a negative influence on patient safety silence $(\beta=-.23, p=.002)$. Patient safety motivation had a negative influence on patient safety silence $(\beta=-.33, p=.006)$ whereas patient safety attitude $(\beta$ $=-.10, p=.964)$ did not influence patient safety silence. The model of patient safety silence explained $36.0 \%$ of variances. As such, 9 out of 11 hypotheses were confirmed (Figure 2).

\section{2) Direct, Indirect, and Total Effects}

To identify the significance of the direct, indirect, and total effects between the variables, bootstrapping method was used in this study. The results are presented in Table 2 . The organizational culture perceived by nurses had direct effects on patient safety motivation $(\beta=.26, p=.003)$ and patient safety attitude $(\beta=.43, p<.001)$; it had no direct effect on patient safety silence but had an indirect effect on patient safety silence $(\beta=.18, p=.028)$ with patient safety motivation as a mediating variable. RN-MD collaboration had direct effects on patient safety motivation $(\beta=.33, p<$ $.001)$, patient safety attitude $(\beta=.35, p<.001)$, and patient safety silence $(\beta=-.17, p=.026)$ and an indirect effect on patient safety silence $(\beta=-.11, p=.008)$ with patient safety mo- tivation as a mediating variable. Supervisory trust had direct effects on patient safety motivation $(\beta=.26, p<.001)$, patient safety attitude $(\beta=.12, p=.036)$, and patient safety silence $(\beta=-.23, p=.002)$ and an indirect effect on patient safety silence $(\beta=-.08, p=.028)$ with patient safety motivation as a mediating variable. Patient safety motivation had a direct effect on patient safety silence $(\beta=-.33, p=.006)$.

\section{DISCUSSION}

The aim of this study was to identify the influence of organizational culture perceived by nurses, RN-MD collaboration, and supervisory trust on patient safety silence mediated by factors of patient safety motivation and patient safety attitude in order to provide theoretical foundations for developing strategies to reject the patient safety silence of nurses. Discussions focused on this objective.

First, the organizational culture perceived by nurses directly influenced patient safety motivation and patient safety attitude, and this finding is in line with that of an existing study on the nurses and doctors at an oncology ward in Switzerland [13]. Most safety accidents tend to be due to problems associated with the hospital organization and its systems rather than the individual faults of medical professionals. For hospitals to prevent medical errors and secure patient safety, it is important to create an organizational culture in which the members of the organization can actively respond to patient safety [28]. Particularly, as the organizational culture is the driving force behind incentivizing specific behavior of its members, the results of this study reconfirmed that the organizational atmosphere of cooperation between its members leads to continuous interest and incentivization towards patient safety for its members [12]. As such, for the managers in hospitals to re- 


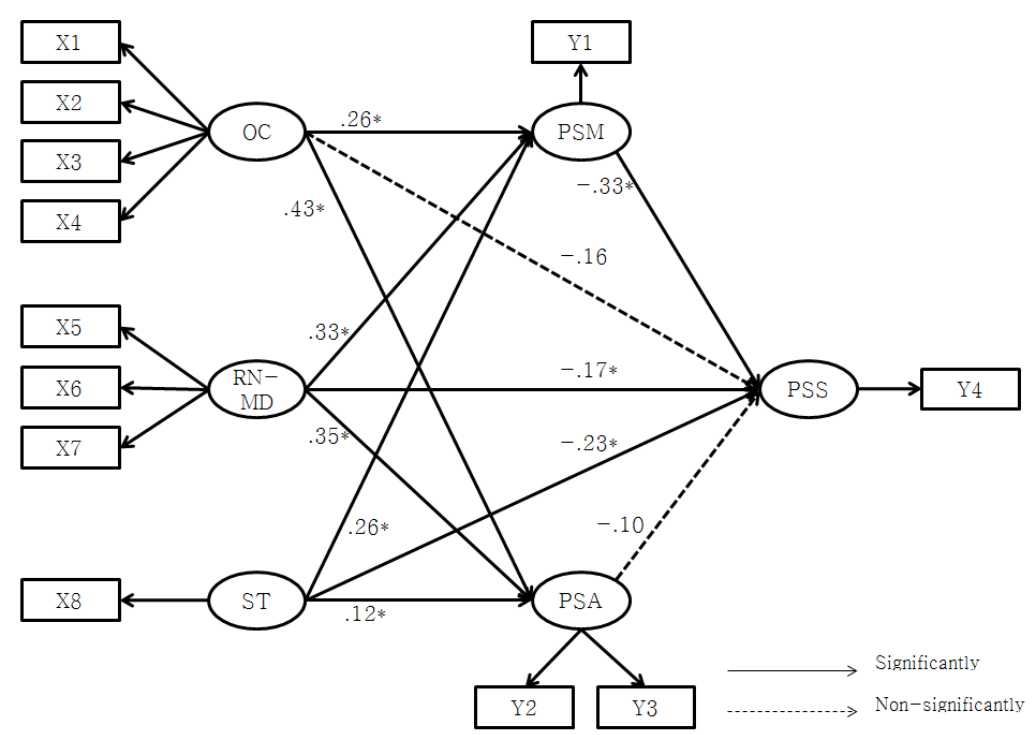

$\mathrm{OC}=$ Organizational culture; $\mathrm{RN}-\mathrm{MD}=\mathrm{RN}-\mathrm{MD}$ collaboration; $\mathrm{ST}=$ Supervisory trust; $\mathrm{PSM}=$ Patient safety motivation; PSA= Patient safety attitude; PSS=Patient safety silence; $X 1=$ Innovative organizational culture; $X 2=$ Relational organizational culture; $X 3=$ Work-oriented organizational culture; $X 4=$ Hierarchical organizational culture; $X 5=S h a r i n g$ of patient's information; $X 6=$ Decision making on the treatment; $\mathrm{X} 7=\mathrm{RN}-\mathrm{MD}$ relationship; $\mathrm{X} 8=$ Supervisory trust; $\mathrm{Y} 1=$ Patient safety motivation; $\mathrm{Y} 2=$ Teamwork; $\mathrm{Y} 3=$ Safety; $\mathrm{Y} 4=$ Patient safety silence; ${ }^{*} p<.05$.

Figure 2. Path diagram for the hypothetical model.

ject behavior related to patient safety silence by nurses, it is important for them to build a positive organizational culture that encourages autonomous reporting and prevention for patient safety. However, in this study, no direct effects of organizational culture on patient safety silence were found but instead an indirect effect on patient safety silence through patient safety motivation as a mediator was found. Based on such findings, it can be concluded that strategies to enhance incentivization for patient safety through strengthening a positive organizational culture appear necessary to reject patient safety silence of nurses.

Second, RN-MD collaboration was found to influence patient safety motivation and patient safety attitude directly. These results can be considered to reconfirm the organizational silence theory presented by Morrison, who also proposed that an atmosphere of cooperation is necessary to effectively prevent patient safety accidents [12]. Particularly, as nurses and doctors play central roles in the recovery of patients' health and their cooperation lead to higher communication accuracy, this can become a critical strategy in preventing patient safety accidents and is directly related to patient mortality [29]. Moreover, it was found that RN-MD collaboration directly influences patient safety silence which suggest that the managers of hospitals should construct a cooperation mechanism for nurses and doctors to facilitate clear communication.
Third, the supervisory trust perceived by nurses directly influenced patient safety motivation and patient safety attitude, and this finding is in line with that of an existing study on nurses and doctors in Sweden [15]. If the members of an organization trust their superiors, they believe that the superior has an excellent ability to work, benevolence, and integrity. Even if the members face work stress, they believe that the superior has the ability to appropriately resolve the negative effects that may come from such work stress (ability), utilize the ability for the members (benevolence), and trust that the superior will direct them to the right path (integrity)[30]. This trust in supervisors has a similar influence on patient safety silence. If nurses have a high degree of trust in their superiors, they maintain an attitude of being continuously interested in acting and striving towards preventing patient safety accidents. In this process, they discuss any difficulties with their superiors, reject patient safety silence. As such, to raise the incentives for patient safety for nurses and reduce actions related to patient safety silence, it is important for hospitals to educate their managers so that the members of their team can raise the degree of trust they have towards their superiors as well as provide a diverse range of programs and education to improve the level of trust between the members and their superiors.

Fourth, it was found that while patient safety motiva- 
tion had a direct influence on patient safety silence, patient safety attitude did not influence patient safety silence. There is a lack of relevant existing literature, which makes accurate comparisons difficult However, Martinez and his colleagues [14] emphasized that not only clinical nurses but also doctors must reject silence regarding patient safety and that the silence of medical professionals towards patient safety begins from indifference, and these results appear to be in line with their emphasis on incentivization of medical professionals regarding patient safety. These results indicate that patient safety motivation is a state in which individual attempts to act safely and emphasizes safety, and it is a determining factor in the direction, degree, and duration of actions by members towards patient safety [25]; as such, patient safety motivation of nurses has a direct influence on patient safety silence. Therefore, in order to reject the patient safety silence of nurses, it is important to confirm the various factors that can incentivize the patient safety motivation of nurses and provide educational programs that focus on these factors.

Lastly, the results of this study showed that the organizational culture, the RN-MD collaboration, and supervisory trust had indirect effects on the patient safety silence of nurses mediated by patient safety motivation and attitude. The results are in line with that of the study by Ullstrom et al.[15], in which MDs and RNs in Sweden were studied, and also that of Schwappach and Gehring [13], who studied MDs and RNs working in an oncology ward in Switzerland. Results of this study suggest that active intervention for the motivation and attitude, psychological aspects of nurses, as well as the organizational atmosphere and the importance of interpersonal relationship toward the patient safety silence of nurses play the key role in avoiding patient safety silence. Consequently, the silence of members in an organization is a multidimensional problem rather than a one-dimensional problem. The results of this study imply that the phenomenon cannot be improved unless the structural component of an organization and individual aspects are considered simultaneously. Moreover, when taking into account Morrison's theory [9], nurses' lack of interest and motivation attention could be the direct causes of the patient safety silence of nurses. However, it is implied that a continuous interest and motivation for patient safety among members requires an institutional tool which facilitates collaboration of members in a hospital and supervisors and co-workers which leads to an effective response to a patient safety accident [12]. Therefore, it is necessary to develop and provide education and programs that can improve the motivation and attitude of nurses toward patient safety in or- der to avoid the patient safety silence of nurses. It is important that the training and education involve improvement of the organizational culture, the RN-MD collaboration, and supervisory trust.

However, it also seems necessary to confirm the various factors related to patient safety silence in order to develop patient safety silence prevention programs for nurses based on such findings. Furthermore, future studies are needed to examine the factors affecting patient safety silence of nurses according to the hospital environment and diverse hospital levels and to improve the hospital environment accordingly.

\section{CONCLUSION}

The authors of this study sought to develop a hypothetical model of organizational culture perceived by nurses, RN-MD collaboration, supervisory trust, patient safety motivation, patient safety attitude, and patient safety silence and to verify the model. The theoretical implication of this study is related to the setting of the hypothetical model of patient safety silence of nurses and the verification of the goodness of fit of the model and study hypotheses which provide basic data on patient safety silence, which in turn is directly related to patient health. The practical importance of this study is that the results provide the baseline data for providing education and developing programs that can avoid patient safety silence among nurses. Moreover, the educational importance of this study is that it provides college students majoring in nursing with educational material related to patient safety silence and conveys the importance of patient safety silence. However, in order to avoid patient safety silence among nurses, it is necessary to develop programs that can be practically applied to clinical nurses based on the results of this study.

\section{REFERENCES}

1. Im SB, Kim SA, Kim SJ, Lee S, Hyun MS, Shin SH, et al. Psychiatric-mental health nursing. 7th ed. Paju: Soomoonsa; 2017.

2. Bae SH, Park JS. Development and evaluation of extracurricular coaching programs for improving communication skills and leadership among nursing students. The Journal of Korean Academic Society of Nursing Education. 2015;21(2):202-214. https://doi.org/10.5977/jkasne.2015.21.2.202

3. Jo EJ, Kang JM, Lim KM. Influence of clinical practice stress and stress coping type on self-leadership of nursing student. Journal of the Korea Academia-Industrial cooperation Society. 2017;18(3):94-104. https://doi.org/10.5762/KAIS.2017.18.3.94

4. Park JH, Jo GY. Predictors of subjectives happiness for male 
nursing students. Journal of Korean Data and Information Science Society. 2016;27(2):511-522.

https://doi.org/10.7465/jkdi.2016.27.2.511

5. Lee ES. Impact of life stress on depression, subjective well-being and psychological well-being in nursing students: mediation effects of coping. Journal of the Korea Academia-Industrial cooperation Society. 2017;18(1):55-65. https://doi.org/10.5762/KAIS.2017.18.1.55

6. Kim JH, Jeon HO. Factors influencing subjective happiness in nursing students: Focused on psycho-social factors. Journal of Korean Academy of Psychiatric and Mental Health Nursing. 2016;25(2):123-132.

https://doi.org/10.12934/jkpmhn.2016.25.2.123

7. Brajsa-Zganec A, Lipovcan LK, Ivanovic D, Larsen ZP. Wellbeing of nursing students: Role of affect regulation, self-esteem, family cohesion and social support. The Open Public Health Journal. 2017;10:69-79. https://doi.org/10.2174/1874944501710010069

8. Jun WH, Cha KS, Lee KL. Factors affecting subjective well-being in nursing students. The Journal of Korean Academic Society of Nursing Education. 2015;21(2):276-284.

https://doi.org/10.5977/jkasne.2015.21.2.276

9. Argyle M, Martin M, Crossland J. Happiness as a function of personality and social encounters. In: Forgas JP, Innes JM, editors. Recent Advances in Social Psychology. Netherlands: Elsevier; 1989. p. 189-203.

10. Lyubomirsky S, King L, Diener E. The benefits of frequent positive affect: Does happiness lead to success?. Psychological Bulletin. 2005;131(6):803-855.

https://doi.org/10.1037/0033-2909.131.6.803

11. Ryan RM, Deci EL. Self-determination theory and the facilitation of intrinsic motivation, social development, and wellbeing. American Psychologist. 2000;55(1):68-78. https://doi.org/10.1037/0003-066X.55.1.68

12. Jo MJ. The factors related to happiness among nursing students. The Journal of Korean Academic Society of Nursing Education. 2016;22(2):182-190.

https://doi.org/10.5977/jkasne.2016.22.2.182

13. Park HS, Han JY. Self-leadership, critical thinking disposition, satisfaction of clinical practice and clinical practice competency of nursing students. Journal of the Korean Data and Information Science Society. 2015;26(3):695-706. https://doi.org/10.7465/jkdi.2015.26.3.695

14. Ha JH, Jang YJ. The relations of perfectionism to subjective well-being and perceived social support giving among college students: Received social support as a mediator. The Korean Journal of Counseling and Psychotherapy. 2011;23(2):427-449.

15. Park HJ. Correlations among nursing professionalism, critical thinking disposition and self-leadership in nursing students. Journal of Korean Academic Society of Nursing Education.
2015;21(2):227-236.

https://doi.org/10.5977/jkasne.2015.21.2.227

16. Lee JY. A study on nurse image, professional self-concept and organizational commitment of nursing student. Journal of the Korea Academia-Industrial cooperation Society. 2017;18(3): 284-295. https://doi.org/10.5762/KAIS.2017.18.3.284

17. Kwon YH, Kwag OG. Validation of a path model on clinical practice competency of the nursing students. Journal of the Korea Academia-Industrial cooperation Society. 2012;13(10): 4572-4581. https://doi.org/10.5762/KAIS.2012.13.10.4572

18. Lee MS, Ha YO. Critical thinking disposition, professional selfconcept and satisfaction of clinical practice in nursing students. Journal of the Korea Academia-Industrial cooperation Society. 2015;16(2):8738-8744.

https://doi.org/10.5762/KAIS.2015.16.12.8738

19. Ji EM, Lee JH. Influencing factors of satisfaction for clinical practice in nursing students. Journal of the Korean Data Analysis Society. 2014;16(2):1125-1140.

20. Tyczkowski B. Vandentyles among nurse managers. Nursing Administration Quarterly. 2015;39(2):172-180. https://doi.org/10.1097/NAQ.0000000000000094

21. Shin KA, Cho BH. Professional self-concept, critical thinking disposition and clinical competence in nursing students. Journal of Korean Academy of Fundamentals of Nursing. 2012;19 (1):46-56.

22. Hills P, Argyle M. The Oxford happiness questionnaire: A compact scale for the measurement of psychological wellbeing. Personality and Individual Differences. 2002;33(7):1073-1082. https://doi.org/10.1016/S0191-8869(01)00213-6

23. Choi YW, Lee SJ. The influence of individual differences in emotional awareness on mental health of college students. Korean Journal of Health Psychology. 2004;9(4):887-901.

24. Jeong HU, Park BG, Choi SW, Kang IG. A study to develop the diagnostic scale of leadership. Seoul: Korean Educational Development Institute; 2003.

25. Yoon J. Development of an instrument for the measurement of critical thinking disposition: In nursing [dissertation]. Seoul: The Catholic University of Korea; 2004.

26. Arthur D. Measuring the professional self-concept of nurses: A critical review. Journal of Advanced Nursing. 1992;17(6):712719. https://doi.org/10.1111/j.1365-2648.1992.tb01969.x

27. Sohng KY, Noh CH. An analytical study of the professional self-concept of hospital nurses in Korea. Journal of Nurses Academic Society. 1996;26(1):94-106.

https://doi.org/10.4040/jnas.1996.26.1.94

28. Lee KS, Ahn SA. Effects of nurses image, psychological wellbeing and career decision level on career preparation behavior in nursing students. Asia-pacific Journal of Multimedia Services Convergent with Art, Humanities and Sociology. 2017;7 (2):809-821. https://doi.org/10.14257/AJMAHS.2017.02.15 